\title{
Intermediate steps towards the achievement of an official romanian translation of the Fugl-Meyer assesment scale specific forms
}

\section{CONSTANTIN Elena ${ }^{1}$, OPREA (MANDU) Mihaela ${ }^{1}$, FRUNZA Andreea ${ }^{1}$, BADIU Cristinel Dumitru ${ }^{1,2}$, OPREA Cosmin Daniel ${ }^{3}$, ANGHELESCU Aurelian ${ }^{1,2}$, POPESCU Cristina ${ }^{1}$, ONOSE Gelu ${ }^{1,2}$}

\author{
Corresponding author: Popescu Cristina, E-mail: cristina popescu recuperare@yahoo.com
}

1.The Teaching Emergency Hospital "Bagdasar-Arseni” (TEHBA), Bucharest, Romania 2.The University of Medicine and Pharmacy "Carol Davila" (UMPCD), Bucharest, Romania

\begin{abstract}
Introduction. The Fugl-Meyer assessment scale for the evaluation of neuro-sensory-motor deficits after stroke represents, by completeness and adequate folding, both conceptually and methodologically, on the physio pathological and clinical-evolutionary reality of disability in this type of pathology, a widely used quantification tool for international level and well appreciated in many works in profile literature.

Materials and methods. From the desire to implement the scale within the neurorehabilitation units in our country, some correspondence with the right holders of the use of the scale within the University of Gothenburg was initiated in 2019. Subsequently, the group proposed us to carry out an official translation according to an algorithm for achieving the unitary translation, agreed and recommended by the official administrators of the standardized forms of the scale, which will be included on the official website of the respective university along with other translations.

Results. Following the initial steps, a constructive correspondence was maintained with the official administrators of the University of Gothenburg and in accordance with the mutual agreement, we carried out the translation from English into Romanian of the specific forms on the official site. The translation included, at the recommendation of the Gothenberg collective, only the component used for measuring the motor functions for the upper and lower extremities. In addition, Prof. Dr. Roxana Carare was co-opted in the team of. Currently, the confrontation of the translation version of our team with the one made by her (forward from English to Romanian) is underway. Within the confrontation of forward translation, different shades of formulations were found at different levels.

Conclusions. In the later stages, the reverse confrontation from Romanian to English (backward) of the two translated variants is considered. At the same time, the coordinator of the administrators of the scale of the University of Gothenburg, Prof. Dr. Margit Alt Murphy, expressed her availability of assistance at all stages of the translation process.
\end{abstract}

Keywords: Fugl-Meyer scale, stroke, assesment, hemiparetic patients, rehabilitation,

\section{Introduction}

Cerebrovascular accidents represent a major cause of morbidity, mortality and disability in the adult population. After a stroke, many patients remain with a serious deficit including motor, sensory and balance, which affect their quality of life. To quantify these deficits, various tools have been created, such as the Fugl-Meyer assessment scale (FMA). It was elaborated in 1975 by Fugl Meyer and his colleagues, who observed the fact that there was a lack of exhaustive quantification of recovery progress in patients who suffered a stroke. The Fugl-Meyer assessment scale for the evaluation of neuro-sensory-motor deficits after stroke is a valid, reliable, responsive, and widely used standardized observational rating scale with ordinal data that assesses the reflex, sensorimotor, balance, joint pain and joint motion impairment. It represents, by completeness and adequate folding, both conceptually and methodologically, on the physio pathological and clinical-evolutionary reality of disability in this type of pathology, a widely used quantification tool for international level and well appreciated in many works in profile literature.(2)

MATERIALS \& METHODS: This paper is a try to extend the implementation of this scale in the inner neurorehabilitation units considering literature related resources (some updated too), aiming to supplement the assessment tools bundle to be availed; this would facilitate more complete evaluated cases in clinical studies. The scale comprises five domains: motor functioning (in the upper extremities maximum 66 points - upper extremity (0-36), wrist (0-10), hand (0-14), coordination/speed (0-6); in the lower extremity maximum 34 points for motor functioning: 0-28 and for coordination/speed:0-6); sensory functioning (maximum 24 points), balance (maximum 14 points), joint range of motion (maximum 44 points, but the less are the points identified aferent to this item, the better the clinical functional statement of the assesed patient), joint pain (maximum 44 points). The rating is based on direct functional quantified observation of the motor performance at each item using a 3-point ordinal scale 
$(0=$ cannot perform, $1=$ performs partially, and $2=$ performs fully). A particularity of this scale refers to the positive relation between functionality and its partial and global scores, and considers - very applied regarding the relation between reflexes evolutiv status and the one of the motor recovery, including passing trough different standardised synergies patterns dinamics within the rehabilitation process. $(1,2,4,5)$ The maximum score that can be achieved is 226 points. The time to be performed is about 45 minutes. $(1,2,5,6)$

Aiming to implement the scale within the neurorehabilitation units in our country, a correspondence with the right holders of the use of the scale within the University of Gothenburg was initiated in 2019. Subsequently, the group proposed us to carry out an official translation according to an algorithm for achieving the unitary translation, agreed and recommended by the official administrators of the standardized forms of the scale, which will be included on the official website of the respective university along with other translations.

RESULTS: Considering on one hand its above mentioned qualities, but on the other its rather chronophagic paradigm, we proposed, in a previous work, a splitting of its achievement in each tested by FMA patient of its specific measurement items between doctors and licensed kinesio-therapists - preliminary specific training based. In this purpose, we have initiated a detailed correspondence with the international professionals in charge of FMA use.Following the correspondence with the holders of the right of use and with those who drafted the standard evaluation form, although the use is free, we received, on one hand, the acceptance of using this ladder under the conditions requested by the respective group of the University of Gothenburg, and we understood that this scale can also be used by being broken down into components. Specifically, the balance section can be opt out (fact even recommended by the respective group), considering that grids or scales for this assessments such as Berg is preferable, which is why in their standardized forms it does not appear in the Balance section (existing in its original form since 1975). In the same conceptual trend we consider, also out of the need to save time to eliminate as many of the redundancies, that the Pain component can also be given up for evaluation, existing a much simpler and specific scale: VAS - Visual Analogue Scale (including with the variant VRS - Verbal Rating Scale). Following the initial steps, a constructive correspondence was maintained with the official administrators of the University of Gothenburg and in accordance with the mutual agreement, we carried out the translation from English into Romanian of the specific forms on the official site. The translation included, at the recommendation of the Gothenberg collective, only the component used for measuring the motor functions for the upper and lower extremities. In addition, Prof. Dr. Roxana Carare was co-opted in the team of. Currently, the confrontation of the translation version of our team with the one made by her (forward from English to Romanian) was realized. Within the confrontation of forward translation, different shades of formulations were found at different levels, due to the transcultural differences, resulting in a pre-final version of translation. Then, according to the quite standardized translation and transcultural linguistic semantic adaptation of different assessment tools, we have proceed to the backward translation - than achieved by a specialized in translation company. Currently our complex related endeavor is on going, i.e. we are now fulfilling the affective final version of the translation into Romanian of the FMA and afterword we shall promptly process to the enrollment according to all the required Bioethics standards - of a lot and compassing (10-15 patients) in orther to make a connected validation of the FMA Romanian version clinical study.

In purpose to overall accomplish this complex work, we have also elaborated a guiding synopsis/ design of it:

Study synopsis:

I. Introduction. Background - including with the specification of the official administrators of the Fugl-Meyer Assessment (FMA) scale's preliminary approval, and further: proposal to initiate this endeavor and subsequent related counseling and support

II. Objectives: transcultural translation and linguistic-semantic adaptation into Romanian of the FMA scale

\section{Materials and Methods}

- Fulfillment of recommended - including for previous such endeavors - steps/ procedures to achieve the translation into Romanian of the FMA scale, with its transcultural, semantic adaptation:

- Forward translation into Romanian from English, by two independent translators good English speakers, reviewed by a quasi-equal Romanian and English speaker (living and working in the UK for about 25 years), and re-reviewed by the expert group, among the authors - thus resulting in the first into Romanian translation version of the FMA

- Backward translation into English from Romanian, by an independent official translator - a prestigious company specialized in translations

- $2^{\text {nd }}$ revision of the first Romanian version of the FMA - including with linguistic-semantic check and adaptations - through crossed analysis by the expert group, among the authors - including with another independent quasi-equal Romanian and English speaker (living and working in the UK for about 25 years) - thus resulting in the second into Romanian 
translation version of the FMA - with linguisticsemantic adaptations

- Initiation of the validation pilot study on hemiparetic post-stroke patients, entailing an additional preliminary revision (overall the $3^{\text {rd }}$ version) of the into Romanian translation of the FMA - including with the related linguistic-semantic check and adaptations - thus resulting in the final into Romanian translation version of the FMA

\section{- Validation pilot study}

- Enrollment of a lot comprising 15 patients

- Fulfillment of the Bio-Ethics preliminary, rigorous, and complete, related procedures

- Patients selection

- Inclusion criteria: post-stroke subacute, subchronic or chronic - minimum there weeks since the acute cerebro-vascular accident (CVA)/ stroke/ brain attack - hemiparetic patients; $\geq 18$ years old inpatients

- Exclusion criteria: poor/ unsteady general health (including neurological) state, sensory (tactile, proprioceptive - with related balance and coordination incurred by cerebellum damages, too -, eyesight and/or auditory) impairments, marked communication (aphasia with receptive elements) and/or (even mild) cognitive troubles, complete or segmentary absence of (a) $\operatorname{limbs} /(\mathrm{s})$, any other matter that could negatively affect the patient's collaboration to this kind of assessment

- The clinical-functional instruments used to assess the enrolled patients with post-stroke hemiparesis: FMA - the translated into Romanian final version - standardized protocols for the upper extremity (UE) and respectively, for the lower extremity (LE); the modified Rankin scale (mRS - as source of overall disability status in each recruited patient); the Barthel index as reference/ "gold standard" for the concurrent validity testing of/ with the FMA; the Montreal Cognitive Assessment (MoCa https://strokengine.ca/en/

https://strokengine.ca/en/assessments/

https://strokengine.ca/en/assessments/montrealcognitive-assessment-moca/) for the cognitive state assessment

- Quantified evaluation of the enrolled patients including in dynamics - by the above mentioned scales used, has been performed at admission at discharge (after about 4 weeks), through the following test/ re-test approach to assay the interand intra-rater reliability:

- Specifically: each patient will be evaluated, at admission and at discharge, simultaneously (i. e directly by one and indirectly by the other one) by two knower of administering the FMA scale, licensed kinesi (physio) therapists, independently, during two days, consecutively; more precisely, one of them will effectively examine and score the patient through the FMA scale, while the other one will observe this evaluation, and based on the respective observation, will score the FMA scale for the same respective patient, without communication in between the two respective examiners neither at the moment of the assessment, nor later, and their results of the FMA will remain unknown for each of the two assessors. The next day the same examiners - i. e. the two knower of administering the FMA scale, licensed kinesi (physio) therapists - will proceed in the same way, but inversing their roles; it will result thus, on one hand, two scores obtained, for the same patient, through the evaluation of the same licensed kinesi (physio) therapist in two consecutive days (intrarater assay) and also two independent scores obtained, consequent to the assessment of the same patient, obtained by the respective two examinants (inter-rater assay)

- Statistical analysis afferent to the validation processing endeavors/ procedures:

- the Svensson method - especially for paired ordinal data (http://avdic.se/svenssonsmetod.html) preferable for objectifying and quantifying the intraand inter-rater reliability - will be used to determine the 'consensus level $(\mathrm{PA}=$ percentage of agreement) between the first and the second observation (for each rater) and between the two different raters (during the same session) ... estimated for each individual item of the FMA ... disagreement between raters ... evaluated by the Relative Position and the Relative Concentration ... The Relative Position indicates the extent to which the distribution of scores from an assessment is systematically shifted towards higher or lower categories. The Relative Concentration shows whether the scores are more or less concentrated towards the central categories of the scale compared to the other assessment. The Relative Position and the Relative Concentration values can vary from -1 to 1 , where 0 means no difference between raters. Values outside the range between -0.1 and 0.1 were considered as clinically relevant disagreements. The Relative Rank Variation indicates non-systematic disagreement caused by individual variability. A value $<0.1$ means that the difference is negligible. Statistically significant disagreements in Relative Position and the Relative Concentration and Relative Rank Variation were indicated in cases when the $95 \%$ confidence interval that did not include the value zero.' (Cecchi $F$ et al. - Transcultural translation and validation of Fugl-Meyer assessment to Italian. 
DISABILITY

AND

REHABILITATION https://doi.org/10.1080/09638288.2020.1746844)

- 'Intraclass Correlation Coefficient (ICC) for testretest reliability' and respectively, standardized response mean (SRM) to test responsiveness, and respectively, Goodness-of-fit index (GFI)- Roman N et al. (2020). Equal Opportunities for Stroke Survivors' Rehabilitation: A Study on the Validity of the Upper Extremity Fugl-Meyer Assessment Scale Translated and Adapted into Romanian. Medicina (Kaunas, Lithuania), 56(8), 409

and also, respectively,

- (intrinsic/ internal) validity

- sensibility ...

- specificity ...

- test efficiency ...

- (extrinsic/ external) validity

- internal/ construct validity ("verified relationships between dependent and independent variables" https://litfl.com/validity-of-clinical-research/)

- Somers $(95 \%$ confidence interval - c. i.) ...

- Spearman $(95 \%$ c. i.) ...

- a Cronbach $(95 \%$ c. i.) ...

- Kendall $(95 \% \%$ c. i.) ...

- Pearson $(95 \%$ c. i. $)$...

(measurement) validity - 'concurrent validity compares measurements with an outcome at the same time (e.g. a concurrent "gold standard" test result)' https://litfl.com/validity-of-clinical-research/

V. Results - see below

VI. Discussion and Conclusions
CONCLUSIONS: We have a very good correspondence of the holders of the right of use. We are permitted to use the protocols free for non-commercial purpose. Additionally, being strongly impressed by our activity and they have also written: "If you have an official translation of the scale in your language done from the original protocol we are interested to see it and consider posting it officially on our webpage together with other translation."

To be mentioned that the representative of the official holders of the FMA expressed aalso their kind availability to assist us along all our above presented complex academic endeavor.

For the validation clinical study we have chosen to compare FMA with the Barthel Index and the (Modified) Rankin Scale.

References:

1. APTA (2011). Compendium of Instructions for Outcome Measures - StrokEDGE Taskforce. APTA Neurology - pp: 51, 55-67, 70-74

2. Fugl-Meyer AR, Jaasko L, Leyman I, et al. The post-stroke hemiplegic patient. 1. A method for evaluation of physical performance. Scand J Rehabil Med. 1975;7:13-31.

3. https://www.sralab.org/rehabilitationmeasures/fugl-meyer-assessment-motor-recoveryafter-stroke

4. https://neurophys.gu.se/sektioner/kliniskneurovetenskap/forskning/rehab med/fugl-meyer

5. https://strokengine.ca/en/assessments/fugl-meyerassessment-of-sensorimotor-recovery-after-strokefma/

6. https://www.gu.se/neurovetenskap-fysiologi/fuglmeyer-assessment 
FMA-UE PROTOCOL

FUGL-MEYER ASSESSMENT

\section{UPPER EXTREMITY (FMA-UE)}

ID:

Assessment of sensorimotor function

Date:

Examiner

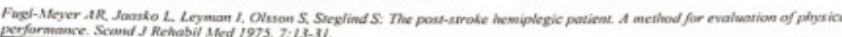

A. UPPER EXTREMITY, sitting position

I. Reflex activity

none can be elicited

\begin{tabular}{|l|l|l|l|}
\hline Extensors: triceps & 0 & 2 \\
\hline
\end{tabular}

\begin{tabular}{|l|l|l|l|}
\hline II. Volitional movernent within synergies, without gravitational help & none & partial & full
\end{tabular}

\begin{tabular}{|l|l|c|c|c|c|}
\hline $\begin{array}{l}\text { Flexor synergy: Hand from } \\
\text { contralateral knee to ipsiateral ear. }\end{array}$ & Shoulder retraction & $\begin{array}{l}\text { relevation } \\
\text { From extensor synergy (shoulder }\end{array}$ & 0 & 1 & 2 \\
\hline
\end{tabular}

adduction/ internal rotation, elbow 1 abduction (90")

extension, forearm pronation) to flexor Elbow fexion

$\begin{array}{lll} & \end{array}$

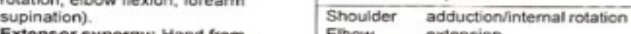

\begin{tabular}{l|l} 
Extensor synergy: Hand from & Elbow extension \\
ipsilateral ear to the contralateral knee & Forearm
\end{tabular}

\begin{tabular}{|l|l|l|l|l|l|l|l|}
\hline III. Volitional movement mixing synergies, without compensation & none & partial & full
\end{tabular} Hand to lumbar spine cannot performior hiand in front of ant sup ilac spine hand on lap

Shoulder flexion $0^{\circ}-90^{\circ}$

elbow at o

hand tontun ât sup tiac spine (withoüt compensat

hand to lumbal, spine ?withoit comperisation maduction at elbowingexion giaring mo

\begin{tabular}{l|l} 
pronation-supination $0^{*}$ & flexion $90^{*}$, no shoulderaboviction or elbow flexfor
\end{tabular}

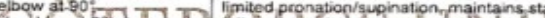

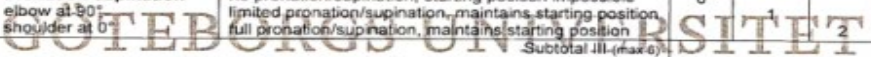

\begin{tabular}{|l|c|c|c|}
\hline IV. Volitional movement with little or no synergy & none & partial & full \\
\hline Shoulder abduction $0-90^{*}$ & immediate supination or elbow flexion & 0 &
\end{tabular}

elbow at $0^{*}$ forearm pronated

Shoulder flexion $90^{\circ}-180^{\circ}$

pronation-supination $0^{*}$

Pronationisupination

exow at $0^{\circ}$
shouider at $30^{\circ}-90^{\circ}$ flexion

supination or elbow flexion during movement

abduction $90^{\circ}$, maintains extension and pronation

immediate abduction or eibow flexion
abduction or elbow fexion during movement

no pronation/supination, startiction pos orition impossible

imited pronation/supination, maintains start position

imited pronation/supination, mantains start position
full pronationsupination, maintains starting position
Subtotal IV (max 6?

V. Normal reflex activity assessed only if full score of 6 points is achieved in

part IV: compare with the unaffected side

biceps, triceps.
finger flexors

2 of 3 reffexes markedly hyperactive or 0 points in part IV
1 reflex markedily hyperactive or at least 2 reflexes lively

Subtotal $V(\max 2)$

Total A (max 36$)$

FMA-UE PROTOCOL

Rehabiltation Medicine, University of Gothenbur

\begin{tabular}{|c|c|c|c|c|}
\hline \multicolumn{2}{|c|}{$\begin{array}{l}\text { B. WRIST support may be provided at the elbow to take or hold the starting } \\
\text { position, no support at wrist, check the passive range of motion prior testing }\end{array}$} & \multirow{2}{*}{\begin{tabular}{c|} 
none \\
0
\end{tabular}} & \multirow{2}{*}{$\begin{array}{c}\text { partial } \\
1\end{array}$} & \multirow{2}{*}{$\begin{array}{c}\text { full } \\
2\end{array}$} \\
\hline $\begin{array}{l}\text { Stability at } 15^{\circ} \text { dorsiflexion } \\
\text { elbow at } 90^{\circ} \text {, forearm pronated } \\
\text { shoulder at } 0^{\circ}\end{array}$ & \begin{tabular}{l|} 
less than $15^{\circ}$ active dorsiflexion \\
dorsiflexion $15^{\circ}$, no resistance tolerated \\
maintains dorsifexion against resistance
\end{tabular} & & & \\
\hline $\begin{array}{l}\text { Repeated dorsifexion / volar flexion } \\
\text { elbow at } 90^{\circ} \text {, forearm pronated } \\
\text { shoulder at } 0^{\circ} \text {, slight finger flexion }\end{array}$ & $\begin{array}{l}\text { Cannot perform volitionally } \\
\text { limited active range of motion } \\
\text { full active range of motion, smoothly }\end{array}$ & 0 & 1 & 2 \\
\hline $\begin{array}{l}\text { Stability at } 15^{\circ} \text { dorsiflexion } \\
\text { elbow at } 0^{*} \text {, forearm pronated } \\
\text { slight shoulder flexion/abduction }\end{array}$ & \begin{tabular}{l|} 
less than $15^{\circ}$ active dorsiffexion \\
dorsiffexion $15^{\circ}$, no resistance tolerated \\
maintains dorsiffexion against resistance
\end{tabular} & 0 & 1 & 2 \\
\hline $\begin{array}{l}\text { Repeated dorsifexion / volar flexion } \\
\text { elbow at } 0^{*} \text {, forearm pronated } \\
\text { slight shoulder flexion/abduction }\end{array}$ & $\begin{array}{l}\text { cannot perform volitionally } \\
\text { limited active range of motion } \\
\text { full active range of motion, smoothly }\end{array}$ & 0 & 1 & 2 \\
\hline $\begin{array}{l}\text { Circumduction } \\
\text { elbow at } 90^{\circ} \text {, forearm pronated } \\
\text { shoulder at } 0^{\circ}\end{array}$ & \begin{tabular}{|l|} 
cannot perform volitionally \\
jerky movement or incomplete \\
complete and smooth circumduction
\end{tabular} & 0 & 1 & 2 \\
\hline & Total $\mathbf{B}_{(\max 10)}$ & & & \\
\hline
\end{tabular}

\begin{tabular}{|l|l|l|l|}
\hline C. HAND support may be provided at the elbow to keep $90^{\circ}$ flexion, no support at & none & partial & full
\end{tabular} \begin{tabular}{|l|l|l|l|l|}
\hline the wrist, compare with unaffected hand, the objects are interposed, active grasp & nono & pantial & fut \\
\hline Mass flexion &
\end{tabular} Mass flexion
from full active or passive extension

\begin{tabular}{l}
$\begin{array}{l}\text { Mass extension } \\
\text { from full active or passive flexion }\end{array}$ \\
\hline GRASP
\end{tabular}

a. Hook grasp
flexion in PIP and DIP (digits II-V).

extension in MCP II-V

b. Thumb adduction

1-st CMC, MCP, IP at $0^{*}$, scrap of paper

between thumb and 2-nd MCP joint

$\begin{aligned} & \text { c. Pincer grasp, opposition } \\ & \text { pulpa of the thumb against the pulpa of } \\ & 20 n d \text { cannot be performed } \\ & \text { can hold pencil but not against tug }\end{aligned}$ 2. Cylinder grasp.
cyllinder shaped object (small can) tug upward, opposition of thumb and

e. Spherical grasp

opposed, tennis ball, tug away

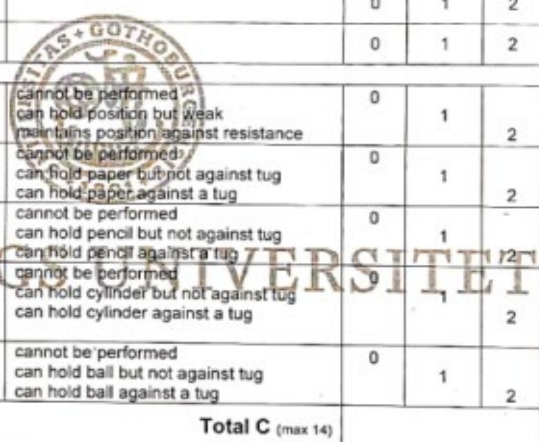

Total C ${ }_{(\max 14)}$

\begin{tabular}{|c|c|c|c|c|}
\hline \multicolumn{2}{|c|}{$\begin{array}{l}\text { D. COORDINATION/SPEED, siting, after one trial with both arms, eyes } \\
\text { closed, tip of the index finger from knee to nose, } 5 \text { times as fast as possible }\end{array}$} & marked & slight & none \\
\hline Tremor & at least 1 completed movement & 0 & 1 & 2 \\
\hline \multirow[t]{2}{*}{$\begin{array}{l}\text { Dysmetria } \\
\text { at least } 1 \text { completed } \\
\text { movement }\end{array}$} & $\begin{array}{l}\text { pronounced or unsystematic } \\
\text { slight and systematic } \\
\text { no dysmetria }\end{array}$ & 0 & 1 & \\
\hline & & $26 \mathrm{~s}$ & $2.5 \mathrm{~s}$ & $<28$ \\
\hline \multirow[t]{2}{*}{$\begin{array}{l}\text { Time } \\
\text { start and end with the } \\
\text { hand on the knee }\end{array}$} & $\begin{array}{l}\text { at least } 6 \text { seconds slower than unaffected side } \\
2-5 \text { seconds slower than unaffected side } \\
\text { less than } 2 \text { seconds difference }\end{array}$ & 0 & 1 & \\
\hline & Total $\mathbf{D}_{(\max 6)}$ & & & \\
\hline
\end{tabular}

FMA-UE PROTOCOL

\begin{tabular}{|c|c|c|c|c|}
\hline \multicolumn{5}{|c|}{ TOTAL A-D $(\max 66)$} \\
\hline \multicolumn{2}{|c|}{$\begin{array}{l}\text { H. SENSATION, upper extremity } \\
\text { eyes closed, compared with the unaflected side }\end{array}$} & \multirow{2}{*}{$\begin{array}{c}\text { anesthesia } \\
0 \\
0\end{array}$} & $\begin{array}{l}\text { hypoesthesia or } \\
\text { dysesthesia }\end{array}$ & \multirow{2}{*}{$\begin{array}{c}\text { normal } \\
2\end{array}$} \\
\hline Light touch & $\begin{array}{l}\text { upper arm, forearm } \\
\text { palmary surface of the hand }\end{array}$ & & $\begin{array}{l}1 \\
1\end{array}$ & \\
\hline & & $\begin{array}{l}\text { less than } 3 / 4 \\
\text { correct or } \\
\text { absence }\end{array}$ & $\begin{array}{c}\text { 3/4 correct or } \\
\text { considerable } \\
\text { difference }\end{array}$ & $\begin{array}{l}\text { correct } 100 \% \text {, } \\
\text { little or no } \\
\text { difference }\end{array}$ \\
\hline $\begin{array}{l}\text { Position } \\
\text { small alterations in the } \\
\text { position }\end{array}$ & $\begin{array}{l}\text { shoulder } \\
\text { elbow } \\
\text { wwist } \\
\text { thumb (IP-joint) }\end{array}$ & $\begin{array}{l}0 \\
0 \\
0 \\
0\end{array}$ & $\begin{array}{l}1 \\
1 \\
1 \\
1 \\
\end{array}$ & $\begin{array}{l}2 \\
2 \\
2 \\
2\end{array}$ \\
\hline & & & Total H (max12) & \\
\hline
\end{tabular}

\begin{tabular}{|c|c|c|c|c|c|c|}
\hline \multicolumn{4}{|c|}{$\begin{array}{l}\text { J. PASSIVE JOINT MOTION, upper extremity, } \\
\text { sitting position, compare with the unaffected side } \\
\text { | only few }\end{array}$} & \multicolumn{3}{|c|}{$\begin{array}{l}\text { J. JOINT PAIN during passive } \\
\text { motion, upper extremity }\end{array}$} \\
\hline & $\begin{array}{l}\text { Only few } \\
\text { degrees } \\
\text { (less than } 10^{*} \text { in } \\
\text { shoulder) }\end{array}$ & decreased & normal & $\begin{array}{l}\text { pronounced pain during } \\
\text { movement or very marked } \\
\text { pain at the end of the } \\
\text { moverment }\end{array}$ & $\begin{array}{l}\text { some } \\
\text { pain }\end{array}$ & $\begin{array}{l}\text { no } \\
\text { pain }\end{array}$ \\
\hline $\begin{array}{l}\text { Shoulder } \\
\text { Flexion }\left(0^{\circ}-180^{\circ}\right) \\
\text { Abduction }\left(0^{\circ}-90^{\circ}\right) \\
\text { External rotation } \\
\text { Internal rotation }\end{array}$ & $\begin{array}{l}0 \\
0 \\
0 \\
0\end{array}$ & & & $\begin{array}{l}0 \\
0 \\
0 \\
0\end{array}$ & $i_{1}^{1}$ & $\begin{array}{l}2 \\
2 \\
2 \\
2\end{array}$ \\
\hline $\begin{array}{l}\text { Elbow } \\
\text { Flexion } \\
\text { Extension } \\
\end{array}$ & : & & & 迿 & $i$ & $\begin{array}{l}2 \\
2\end{array}$ \\
\hline $\begin{array}{l}\text { Forearm } \\
\text { Pronation } \\
\text { Supination }\end{array}$ & $\begin{array}{l}0 \\
0 \\
\end{array}$ & & & $\begin{array}{l}0 \\
0\end{array}$ & 1 & $\frac{2}{2}$ \\
\hline $\begin{array}{l}\text { Wrist } \\
\text { Flexion. } \\
\text { Extensfon } \\
\text { Fingers } \\
\text { Flexion } \\
\text { Extension }\end{array}$ & & 1 & & :- & $\frac{11}{1}$ & $\frac{2^{2}}{\frac{1}{2}}$ \\
\hline \multicolumn{4}{|l|}{ Total (max 24) } & \multicolumn{3}{|l|}{ Total (max 24) } \\
\hline
\end{tabular}

\begin{tabular}{|l|r|}
\hline A. UPPER EXTREMITY & 136 \\
\hline B. WRIST & 110 \\
\hline C. HAND & 114 \\
\hline D. COORDINATION / SPEED & $/ 6$ \\
\hline TOTAL A-D (motor function) & 166 \\
\hline
\end{tabular}

\begin{tabular}{|l|r|}
\hline H. SENSATION & 112 \\
\hline J. PASSIVE JOINT MOTION & 124 \\
\hline J. JOINT PAIN & 124 \\
\hline
\end{tabular}

Fig. 1.Fugl-Meyer assessment scale upper extremity $(3,4)$ 
FMA-LE PROTOCOL

\section{FUGL-MEYER ASSESSMENT \\ LOWER EXTREMITY (FMA-LE) \\ ID \\ Assessment of sensorimotor function Examine}

method for evatuation of phiysical

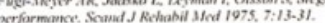

\section{E. LOWER EXTREMITY}

I. Reflex activity, sup

Flexors: knee flexors supine position

Extensors: patellar, achilles (at least one)
Ear

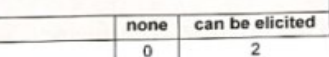

\begin{tabular}{|l|l|l|l|l|}
\hline II. Volitional movement within synergies supine position & none & partial & full
\end{tabular}

\begin{tabular}{l|l|c|c|c|c|}
\hline Flexor synergy: Maximal hip flexion & Hip flexion & 0 & 1 & 2
\end{tabular}

knee and ankle joint (palpate distal tendons to



Extensor synergy: From flexor synergy to the hip Hip extension

plantar flexion. Resistance is applied to ensure adduction active movement, evaluate both movement and Knee extension strength (compare with the unaffected side) 158 . Subtotal II (max 14)

III. Volitional movement mixing synergies

sitting position, knee $10 \mathrm{~cm}$ from the edge of the chair/bed

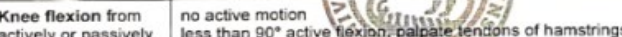

extended knee more than $90^{\circ}$ active héxion

Ankle dorsiflexion $\begin{aligned} & \text { no active motion } \\ & \text { limited dorsiflexion }\end{aligned}$

unaffectéd side, 7 -camplete dorsiflexion

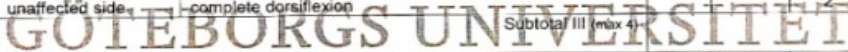
\begin{tabular}{l|c|c|c|}
\hline $\begin{array}{l}\text { IV. Volitional movement with little or no synergy } \\
\text { standing position, hip at } 0^{\circ}\end{array}$ & none & partial & full \\
\hline
\end{tabular} \begin{tabular}{l|l} 
Knee flexion to $90^{\circ}$ & no active motion or immediate, simultaneous hip flexion \\
\hline
\end{tabular}

support is allowed - lat least $90^{\circ} \mathrm{knee}$ flexion without smultaneous hip flexion

$\begin{array}{ll}\text { Ankle dorsiflexion } & \text { no active motion } \\ \text { limited dorsiflexion }\end{array}$

unaffected side

Subtotai IV (max 4$)$

\begin{tabular}{|l|l|l|l|}
\hline V. Normal reflex activity supine position, assessed only if full score of 4 & hyper & lively & normal
\end{tabular} points is achieved in part IV, compare with the unaffected side

\begin{tabular}{l|l} 
Reflex activity & 2 of 3 reflexes markedly hyperactive
\end{tabular}

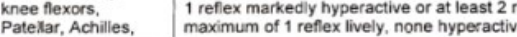

Subtotal V $(\max 2)$

Total $\mathrm{E}(\max 28)$
FMA-LE PROTOCOL

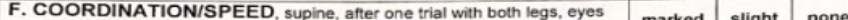
\begin{tabular}{|l|c|c|c|}
\hline closed, heel to knee cap of the opposite leg, 5 times as fast as possible & marked & silght & none \\
\hline Tremor & 0 & 1 & 2 \\
\hline
\end{tabular} Dysmetria

\begin{tabular}{|l|l|c|c|c|}
\hline & $\begin{array}{l}\text { pronounced or unsystematic } \\
\text { slight and systematic } \\
\text { no dysmetria }\end{array}$ & $\geq 6 \mathrm{~s}$ & $\mathbf{2 - 5 s}$ & $<2 \mathrm{~s}$ \\
\hline $\begin{array}{l}\text { Time } \\
\text { start and end with the } \\
\text { hand on the knee }\end{array}$ & $\begin{array}{l}\text { 6 or more seconds slower than unaffected side } \\
2-5 \text { seconds slower than unaffected side } \\
\text { less than 2 seconds difference }\end{array}$ & 0 & 1 & 2 \\
\hline
\end{tabular}
start and end with the Seconds slower than unaffected side

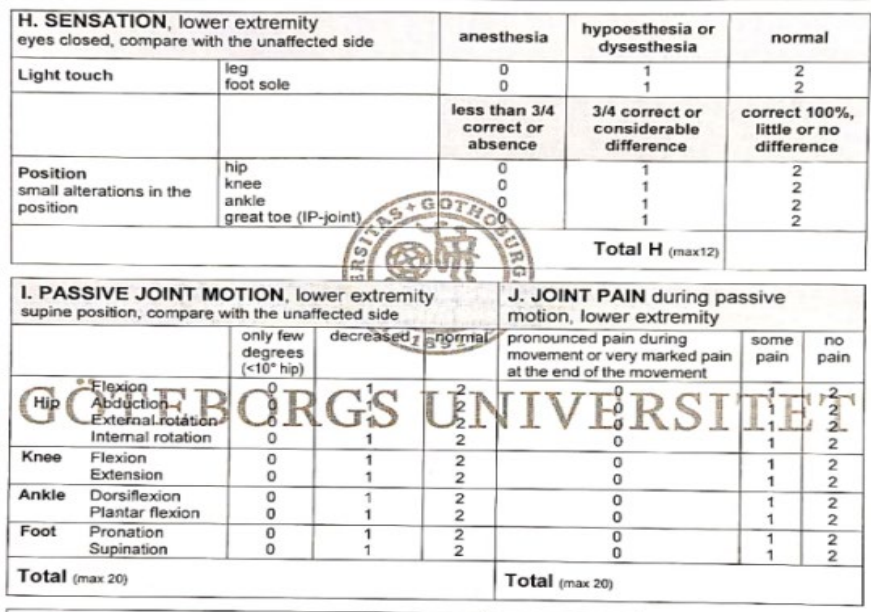

\begin{tabular}{|l|r|}
\hline E. LOWER EXTERMTY & 128 \\
\hline F. COORDINATION / SPEED & $/ 6$ \\
\hline TOTAL E-F (motor function) & 134 \\
\hline
\end{tabular}

H. SENSATION

L. PASSIVE JOINT MOTION

J. JOINT PAIN

Fig. 2. Fugl-Meyer assessment scale lower extremity $(3,4)$

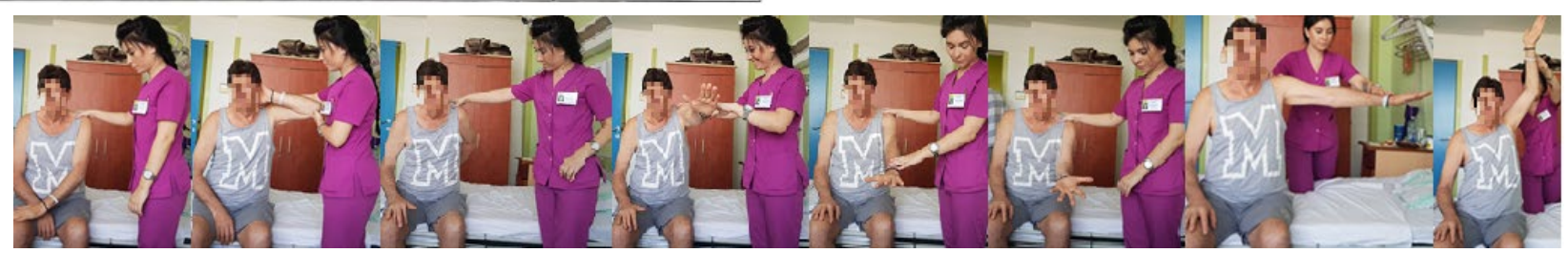

Fig.3. Volitional movement

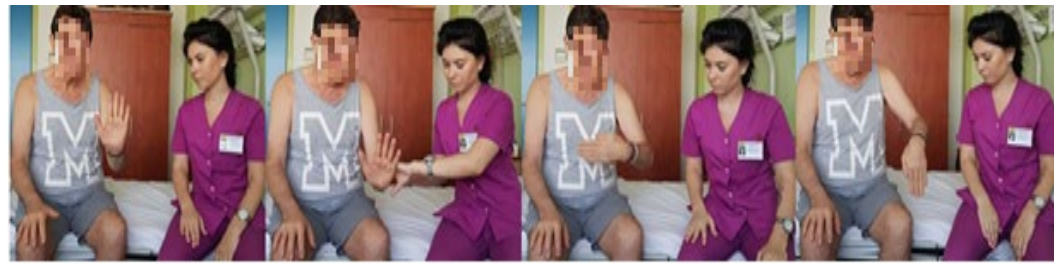

Fig.4. Wrist

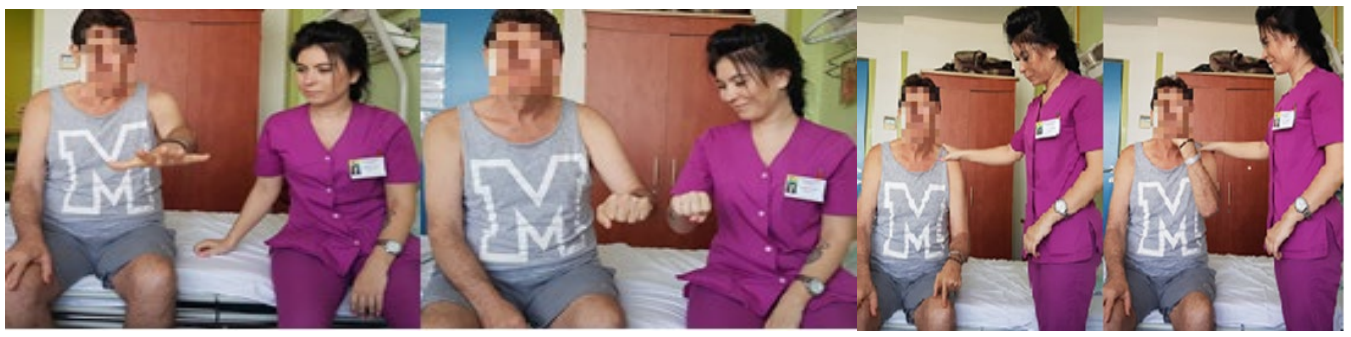

Fig.5. Hand coordination 


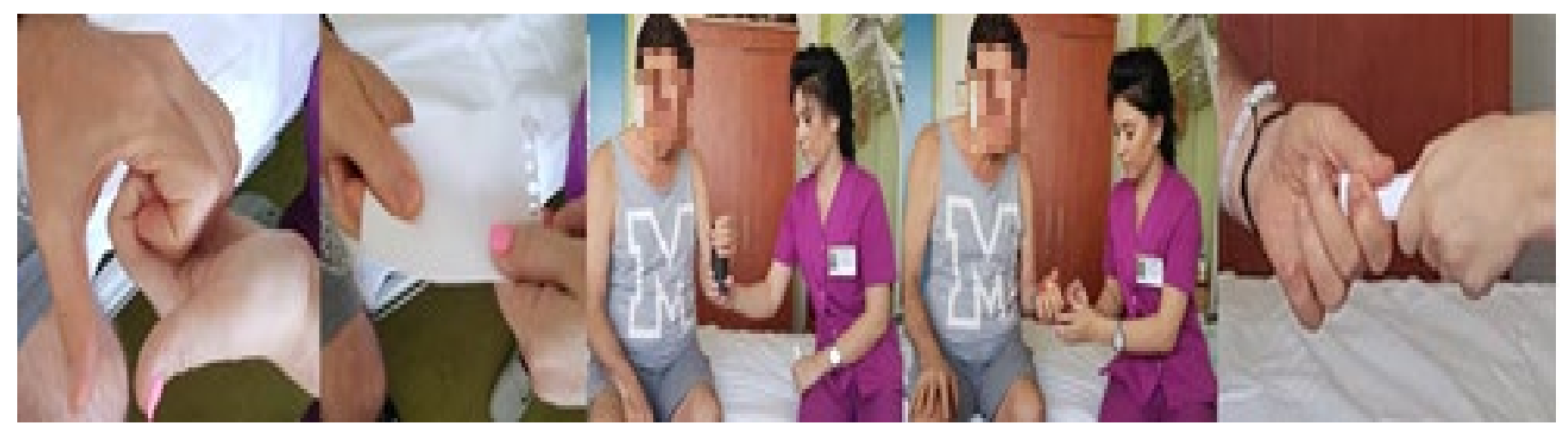

Fig. 6. Hook grasp, thumb adduction, cylinder grasp, spherical grasp, pincer grasp

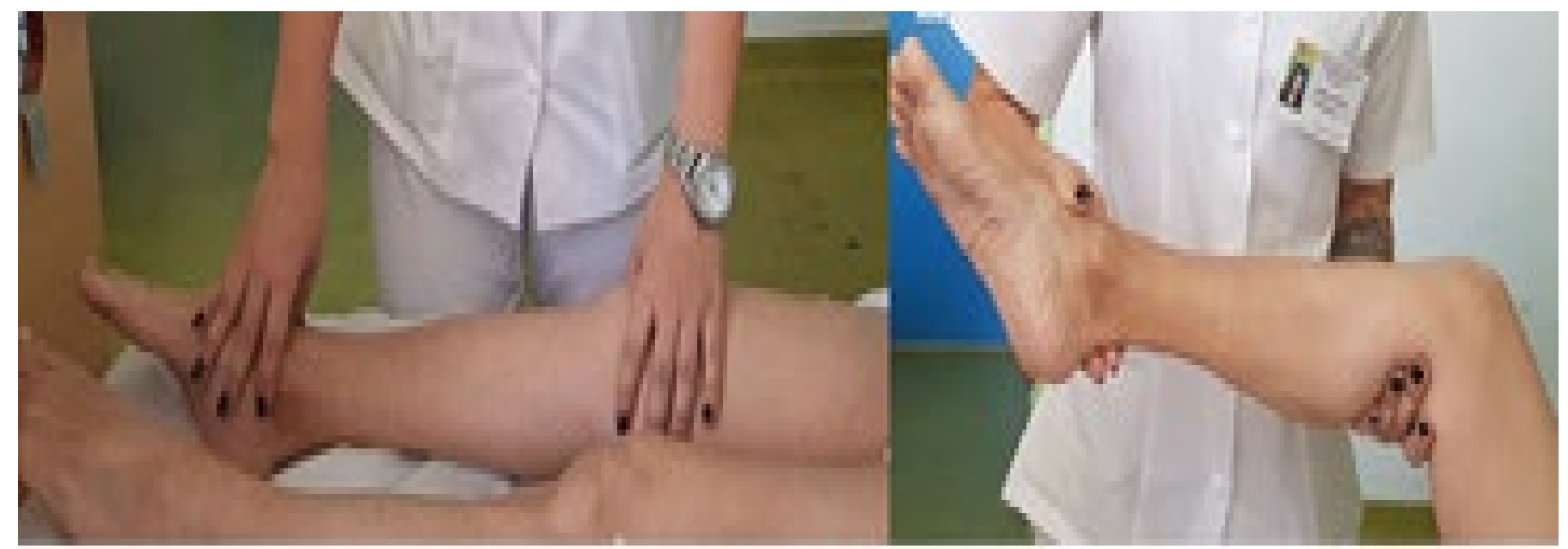

Fig.7. Extensor, flexor synergy

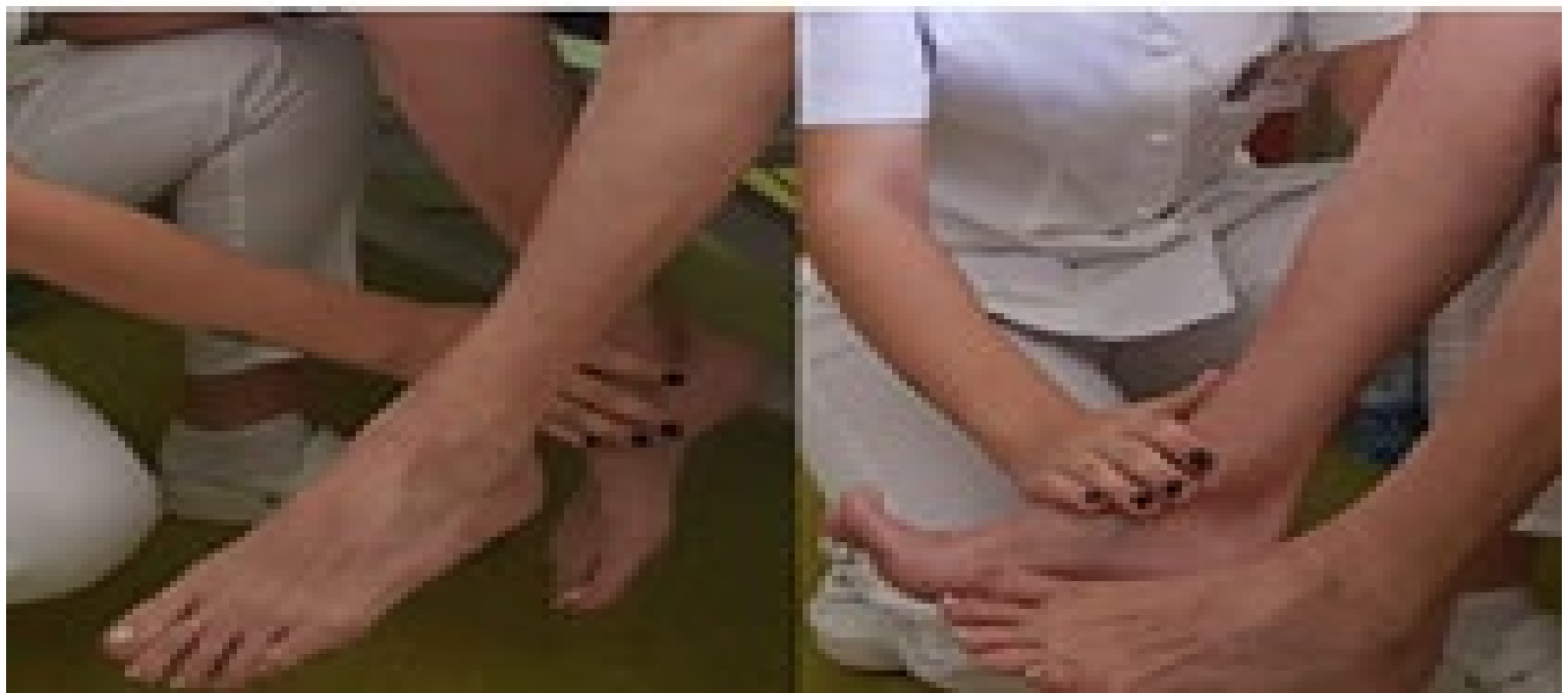

Fig.8. Knee flexion, ankle dorsiflexion 
PROTOCOL de EVALUARE FUGL-MEVER (EFM) Medichs de Reciperare, Universtatea din Gereborr (Gothenturg University - EXTREMITATEA SUPEPHOARA IES EVALUAREA FUGL-MEYER

Dats:

Evaluarea funcţiei senzitivomotorii Examinator:

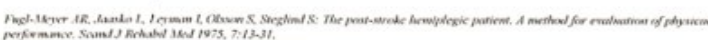

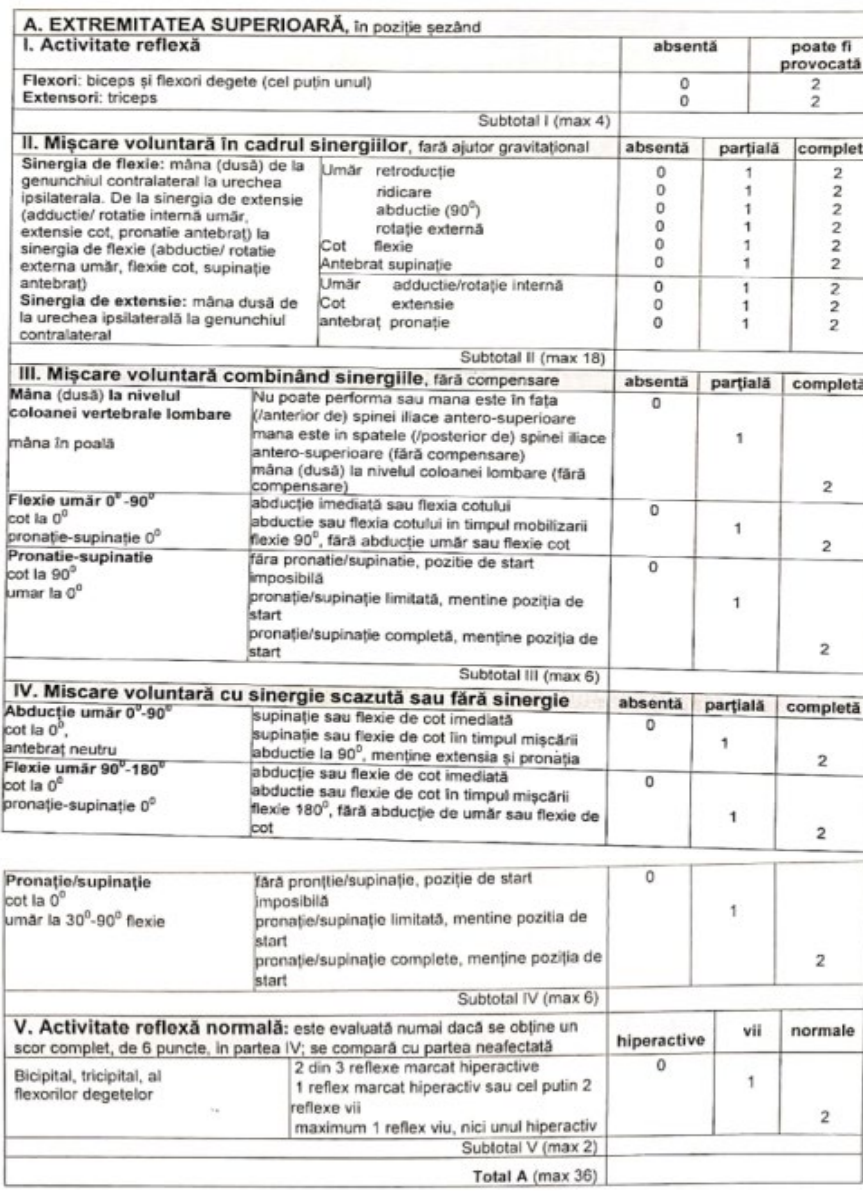

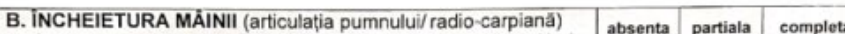
sustinerea acesteia poate fí electuata la cot pentru a lua sau sustine pozitia de de migcare pasiva inainte de testare ot la $90^{\circ}$, antebrat pronat umar la $0^{\circ}$

Dorsiflexie/ flexie volară repetată cot la $90^{\circ}$

antebrat pronar la $0^{\circ}$.

usoara flexie digitala

Stabilitate la $15^{\circ}$ dorsiflexio

cot la $0^{\circ}$, antebrat pronat

soara flexie/abductie umar

Dorsiflexie/hexie volara repetat

$\cot 1 \mathrm{l} 0^{\circ}$.

ușoarà flexie/abductie umăr

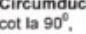

antebrat pronat

umầ la $0^{\circ}$

\begin{tabular}{|c|c|c|c|}
\hline $\begin{array}{l}\text { mai putin de } 15^{\circ} \text {, dorsinexie activä } \\
\text { dorsiflexie } 15^{\circ} \text {, fara rezistenta } \\
\text { mentine dorsifilexia impotriva } \\
\text { rezistentei }\end{array}$ & 0 & 1 & 2 \\
\hline $\begin{array}{l}\text { nu poate performa voluntar } \\
\text { amplitudine de mişcare activà } \\
\text { limitatala } \\
\text { amplitudine de miscare activa } \\
\text { completa, efectuabas lin/ bine }\end{array}$ & 0 & 1 & 2 \\
\hline $\begin{array}{l}\text { mai putin de } 15^{\circ} \text {, dorsiflexie activata } \\
\text { dorsiflexie } 15^{\circ} \text {, tara rezistenta } \\
\text { mentine dorsiflexia impotriva } \\
\text { rezistentei }\end{array}$ & 0 & 1 & 2 \\
\hline $\begin{array}{l}\text { nu poate performa voluntar } \\
\text { amplitudine de misçare activa } \\
\text { imitata } \\
\text { ampitudine de mişcare activa } \\
\text { completá, efectuabila lin/ bine }\end{array}$ & 0 & 1 & 2 \\
\hline $\begin{array}{l}\text { nu poate performa voluntar } \\
\text { miscare sacadatal/ spsmodical } \\
\text { tremurătoare sau incompleta } \\
\text { circumductie completa si linan/ } \\
\text { bine efectuata }\end{array}$ & 0 & 1 & 2 \\
\hline
\end{tabular}

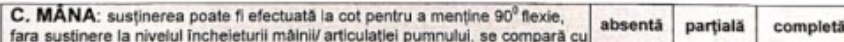
mána neafectatâ, obiectele se interpun, prehensiune/ prindere activă

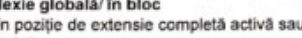

Extensie globala/in bloc

din pozitie de flexie completă activas sau pasiva

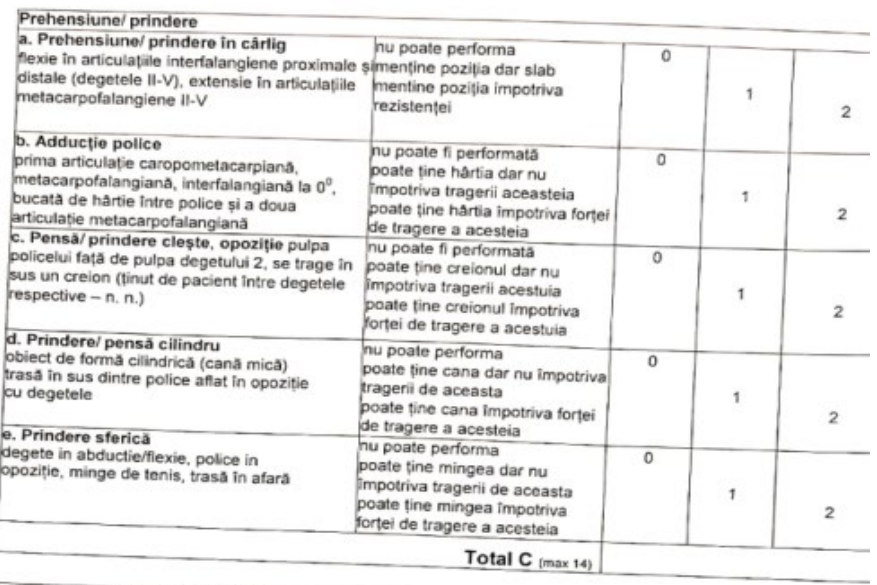

D. COORDONARE/ VITEZÁ, in pozițe sezând, după o testare la $(n \cdot n)$

\begin{tabular}{|c|c|c|c|c|}
\hline \multicolumn{2}{|c|}{ 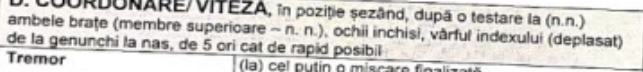 } & marcat & usor & $\begin{array}{c}\text { nu } \\
\text { existá }\end{array}$ \\
\hline & (a) cel puțin o miscare finalizată & 0 & 1 & 2 \\
\hline $\begin{array}{l}\text { ismotrie } \\
\text { cel putin (la) o miscare } \\
\text { completa }\end{array}$ & $\begin{array}{l}\text { pronuntată sau nesistematică } \\
\text { uşoară sau sistematică } \\
\text { târă dismetrie }\end{array}$ & 0 & 1 & 2 \\
\hline & & $26 \mathrm{~s}$ & $2 \%$ & $<2 \mathrm{~s}$ \\
\hline $\begin{array}{l}\text { Timp } \\
\text { incepere si terminare cu } \\
\text { mâna pe genunchi }\end{array}$ & $\begin{array}{l}\text { cu } 6 \text { secunde sau mai mult, mai lent decat in partea } \\
\text { neafectata } \\
\text { cu } 2-5 \text { secunde mai lent decat in partea neafectata } \\
\text { cu mai putin de } 2 \text { secunde diferenta }\end{array}$ & 0 & 1 & \\
\hline & Total D $(\max 6)$ & & & \\
\hline
\end{tabular}

H. SENSIBILITATE, extremitatea superioara, TOTAL A-D $(\max 66)$ |

\begin{tabular}{l|l|l} 
& Thestezio & hipoestezie
\end{tabular}

Atingere uşoara

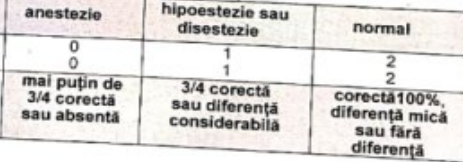

\begin{tabular}{|c|c|c|c|c|}
\hline $\begin{array}{l}\text { Pozitie } \\
\text { alterarari ușoare ale } \\
\text { pozifiei }\end{array}$ & $\begin{array}{l}\text { Jumăr } \\
\text { cot } \\
\text { incheietura máinii (articulatia } \\
\text { pumnuluil radio-carpianá) } \\
\text { police (articulą̧ia } \\
\text { interfalangiana) }\end{array}$ & $\begin{array}{l}0 \\
0 \\
0 \\
0\end{array}$ & $\begin{array}{l}1 \\
1 \\
1 \\
1\end{array}$ & $\begin{array}{l}2 \\
2\end{array}$ \\
\hline
\end{tabular}

1. MOBILITATE ARTICULARÁ PASIVÃ, extremitatea J. DURERE ARTICULARÃ in timpul

\begin{tabular}{|c|c|c|c|c|c|c|}
\hline & $\begin{array}{l}\text { cateva grade } \\
\text { (mai putin de } 10^{\circ} \\
\text { in umarr) }\end{array}$ & scazutâ & normalä & $\begin{array}{c}\text { durere pronuntată in timpul } \\
\text { misccárii sau durere foarte } \\
\text { marcată la sfarșitul mişcârii }\end{array}$ & $\begin{array}{l}\text { durere } \\
\text { ușoară }\end{array}$ & $\begin{array}{c}\text { fară } \\
\text { durere }\end{array}$ \\
\hline \multicolumn{7}{|l|}{ Umăr } \\
\hline Flexie $\left(0^{\circ}-180^{\circ}\right)$ & 0 & 1 & 2 & 0 & 1 & 2 \\
\hline Abductie $\left(0^{\circ}-90^{\circ}\right)$ & 0 & 1 & 2 & 0 & 1 & 2 \\
\hline Rotatie externá & 0 & 1 & 2 & 0 & 1 & \\
\hline Rotatie interna & 0 & 1 & 2 & 0 & & \\
\hline \multicolumn{7}{|l|}{ Cot } \\
\hline $\begin{array}{l}\text { Fexie } \\
\text { Extensie }\end{array}$ & 0 & 1 & 2 & 0 & 1 & $\begin{array}{l}2 \\
2\end{array}$ \\
\hline \multicolumn{7}{|l|}{ Antebrat } \\
\hline $\begin{array}{l}\text { Pronatie } \\
\text { Supinatie }\end{array}$ & $\begin{array}{l}0 \\
0\end{array}$ & 1 & $\begin{array}{l}2 \\
2\end{array}$ & $\begin{array}{l}0 \\
0\end{array}$ & 1 & $\begin{array}{l}2 \\
2\end{array}$ \\
\hline \multicolumn{7}{|l|}{$\begin{array}{l}\text { Incheietura máinii } \\
\text { (articulația pumnuluil }\end{array}$} \\
\hline & & & & 0 & 1 & 2 \\
\hline $\begin{array}{l}\text { Flexie } \\
\text { Extensie }\end{array}$ & $\begin{array}{l}0 \\
0\end{array}$ & 1 & $\begin{array}{l}2 \\
2\end{array}$ & & & \\
\hline \multicolumn{7}{|l|}{ Degete } \\
\hline Flexie & 0 & 1 & 2 & & 1 & 2 \\
\hline Extensic & 0 & 1 & 2 & 0 & 1 & 2 \\
\hline \multicolumn{4}{|l|}{ Total $(\max 24)$} & Total $(\max 24)$ & & \\
\hline
\end{tabular}

\begin{tabular}{|c|c|}
\hline $\begin{array}{l}\text { A. EXTREMITATEA SUPERIOARÃ } \\
\text { B. iNCHEIETURA MÄINIII (articulația pumnulüi' } \\
\text { radio-carpianā) }\end{array}$ & \\
\hline C. MĂNA & 114 \\
\hline D. COORDONARENITEZĀ & 16 \\
\hline TOTAL A-D (functie motorie) & 166 \\
\hline
\end{tabular}

\begin{tabular}{|l|r|}
\hline H. SENSIBILITATE & $/ 12$ \\
\hline L. MOBILITATE ARTICULARĂ PASIVĀ & 124 \\
\hline J. DURERE ARTICULARĀ & 124 \\
\hline
\end{tabular}

Fig. 9. Fugl-Meyer assessment scale translated from English to Romanian (forward)- upper extremity 


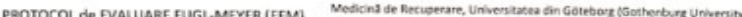

- EXTREMITATEA INFERIOARÃ (EE)

EVALUAREA FUGL-MEYER

EXTREMITATEA INFERIOARĂ (EFM-EI) Data:

Evaluarea funcției senzitivomotorii Examinator:

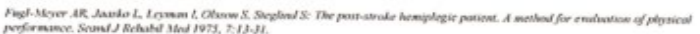

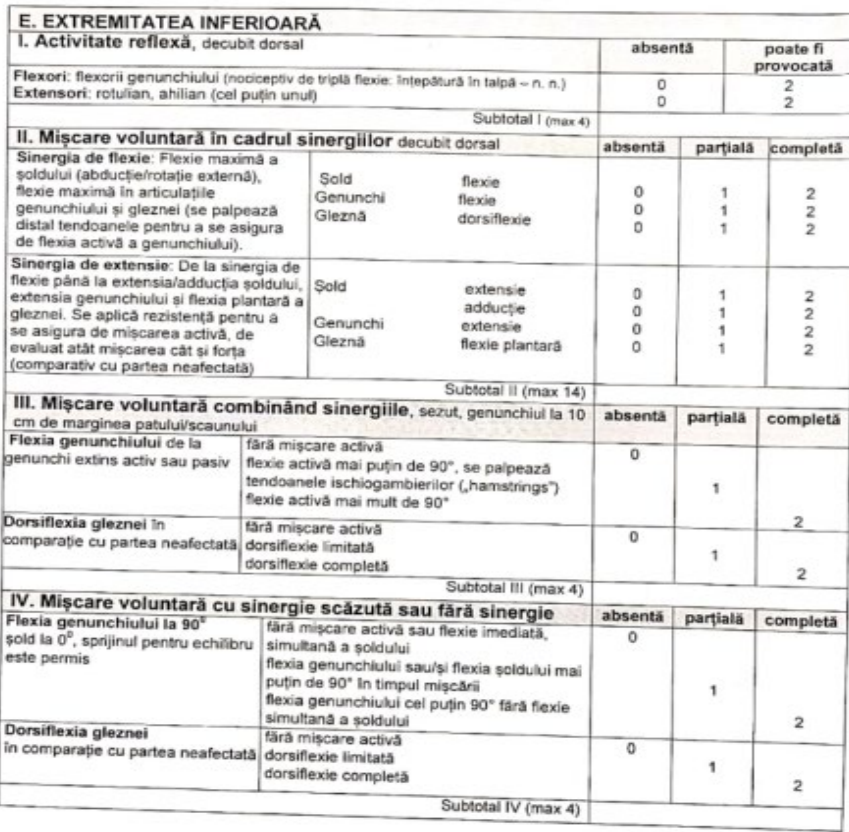

V. Activitate reflexă normală din decubin dorsal ease com

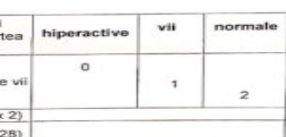

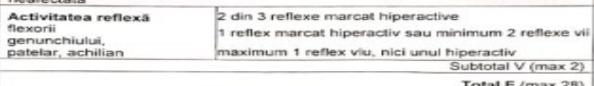

\begin{tabular}{|l|l|l|l|l|}
\hline F. COORDONARE/ VITEZA, in decubit dorsal, dupa o testare cu & absenta & partiala & complota \\
\hline
\end{tabular} ambele picioare, ochili inchist, âling



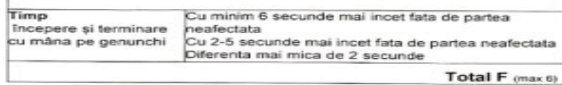

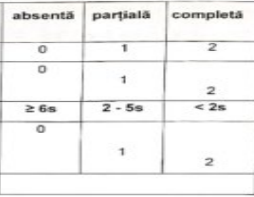

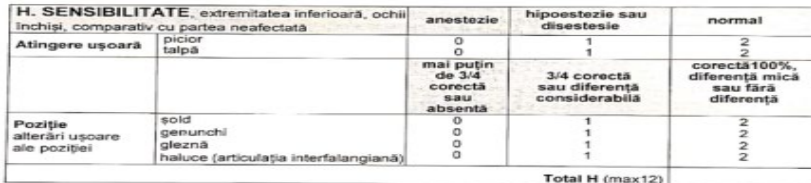

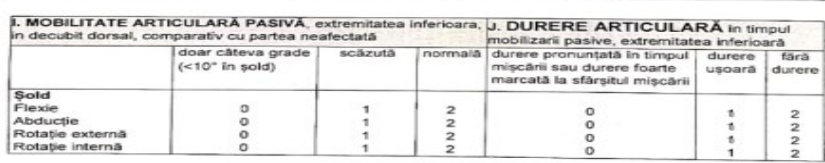

\begin{tabular}{|l|c|c|c|c|c|c|}
\hline $\begin{array}{l}\text { Genunchi } \\
\text { Flexie } \\
\text { Extensie }\end{array}$ & 0 & 1 & 2 & 0 & 1 & 2 \\
Glezna & 0 & 1 & 2 & 0 & 1 & 2 \\
\hline $\begin{array}{l}\text { Dorsiflexie } \\
\text { Flexie plantara }\end{array}$ & 0 & 1 & 2 & 0 & 1 & 2 \\
$\begin{array}{l}\text { Picior } \\
\text { Pronatie } \\
\text { Supinatie }\end{array}$ & 0 & 1 & 2 & 0 & 1 & 2 \\
\hline
\end{tabular}

\begin{tabular}{|l|r|}
\hline E. EXTREMITATEA INFERIOARĀ & 128 \\
\hline F. COORDONARE/VITEZĀ & 16 \\
\hline TOTAL E-F (functie motorie) & 134 \\
\hline
\end{tabular}

\begin{tabular}{|l|r|}
\hline H. SENSIBILITATE & $1 / 2$ \\
\hline L. MOBILTATE ARTICULARA PASIVA & 120 \\
\hline J. DURERE ARTICULARA & 120 \\
\hline
\end{tabular}

Fig. 10. Fugl-Meyer assessment scale translated from English to Romanian (forward)- lower extremity

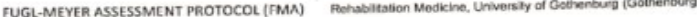

UNWEPSY)

FUGL-MEYER ASSESSMENT Patient Identifier

UPPER EXTREMITY (FMA-UE) Date:

Assessment of sensorimotor function

Examiner:

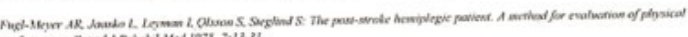

A. UPPER EXTREMITY, in sitting position

- Reflex activity

Flexors: biceps an
Extensors: triceps

Volitional movement within synergies, without graviational aid

Flexor synergy: hand (moved) from Shoulder retraction

From extensor synergy (shoulder
adductionl internal rotation, elbow

extension, forearm pronation) to flexor
external rotation

synergy (shoulder abduction/ external

rotation, elbow
supination)

Forearm supination

Shouider adduction/ internal

Extensor synergy: hand moved from rotaton

the ipsilateral ear to the contralateral Elbow extension
knee

Subtotal II ( $\max 18)$

III. Volitional movement mixing synergies, without compensation

(i) the anterior-superior Ilac spine, the hanter

Hand on the lap

Shoulder flexion $0^{\circ}-90^{\circ}$

Elbow to 0

pronation-supination $0^{\circ}$

pehind (jposterior to) the anterior-superior iliac

spine (without compensation), the hand (moritions
to lumbar spine (without compensation)

immediate abduction or elbow flexion

abduction or elbow flexion during movement

$90^{\circ}$ flexion, no shoulder abduction or elbow

lexion

Pronation-supination ho pronation/supination, impossible starting

imited pronation/supination, maintains the

ull pronation/supination, maintains the starting

position

IV. Volitional movement with little or no synergy

$\begin{array}{ll}\text { Shoulder abduction } 0^{*}-90^{\circ} & \text { Immediate eibow supination or flexion } \\ \text { eibow supination or flexion during movement }\end{array}$

abow supination or flexion during movement

pronation

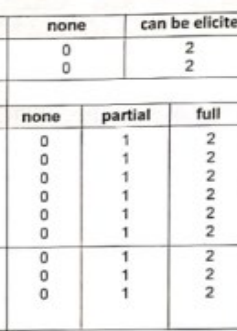

\begin{tabular}{c|c|c|} 
none & partial & full \\
\hline
\end{tabular}

Shoulder flexion $90^{\circ}-180^{\circ}$ Immediate elbow abduction of flexion

elbow to $0^{\circ}$

pronation-supination $0 "$

Pronation/supination

elbow to $0^{\circ}$

shoulder to $30^{\circ} .90^{\circ}$ ferion

thow abduction or flexion during movement fexion $180^{\circ}$, no shoulder abduction or elbow

flexion

no pronation/supination, starting postion

impossble

limiled pronation/supination, maintains starting

position

full pronationsupination, maintains starting

position

Subtotal IV $(\max 6)$

\begin{tabular}{|c|c|c|c|c|}
\hline \multicolumn{2}{|c|}{$\begin{array}{l}\text { V. Normal reflex activity: assessed only if a full score of } 6 \text { points is } \\
\text { achieved in part IV; compare with the unaffected side }\end{array}$} & \multirow{2}{*}{$\begin{array}{c}\text { hyperactiv } \\
e\end{array} \mid$} & \multirow{2}{*}{\begin{tabular}{|c|} 
lively \\
1 \\
\end{tabular}} & \\
\hline $\begin{array}{l}\text { Biciphal, tricipital, of fingers } \\
\text { fexors }\end{array}$ & $\begin{array}{l}2 \text { of } 3 \text { reflexes markedly hyperactive } \\
1 \text { reflex markedly hyperactive or at least } 2 \\
\text { reflexes lively } \\
\text { Maximum of } 1 \text { reflex lively, none } \\
\text { hyperactive }\end{array}$ & & & 2 \\
\hline & Subtotal V (max 2) & & & \\
\hline
\end{tabular}

B. WRIST (fist/radiocarpal joint) support may be provided at the elbow to

take or hoid the starting position, no support at the wrist fist level; check the

passive range of motion before lesting

Stability at $15^{\circ}$ dorsiflexion

elbow at $90^{\circ}$, forearm pronated

shoulder at $0^{\circ}$

less than $15^{\circ}$, active dorsiflexion

dorsifferion $15^{\circ}$, no resistance

dolerated

maintains dorsiflexion against

resistance

Repeated dorsiflexion/volar flexion

elbow at $90^{\circ}$

forearm pronated

shoulder at $0^{\circ}$.

slight finger flexion

Stability at $15^{*}$ dorsiflexion

elbow at $0^{\circ}$, forearm pronated

fight shoulder ferion/abduction

Repeated dorsiflexion/ volar flexion

elbow at $90^{\circ}$

forearm pronated

slight shoulder flexion/abduction

Circumduction

elbow at $90^{\circ}$

forearm pronated

shoulder at $0^{\circ}$

cannot perform volitionaly

limited active range of mot

full active range of motion,

smoothly/ wel realizable

less than $15^{\circ}$, active dorsiflexion

dorsiffexion 15", no resistance

tolerated

maintains dorsiflexion against

resistance

cannot perform volitionally

Imited active range of motio

full active range of motion.

smoothly' well realizable

cannot perform volitionally

movement or incomplete

complete and smoothly/ well don circumduction

Total B $(\max 10)$

\begin{tabular}{|c|c|c|}
\hline none & partial & full \\
\hline 0 & 1 & 2 \\
\hline 0 & 1 & 2 \\
\hline 0 & 1 & 2 \\
\hline 0 & 1 & 2 \\
\hline 0 & & \\
\hline
\end{tabular}



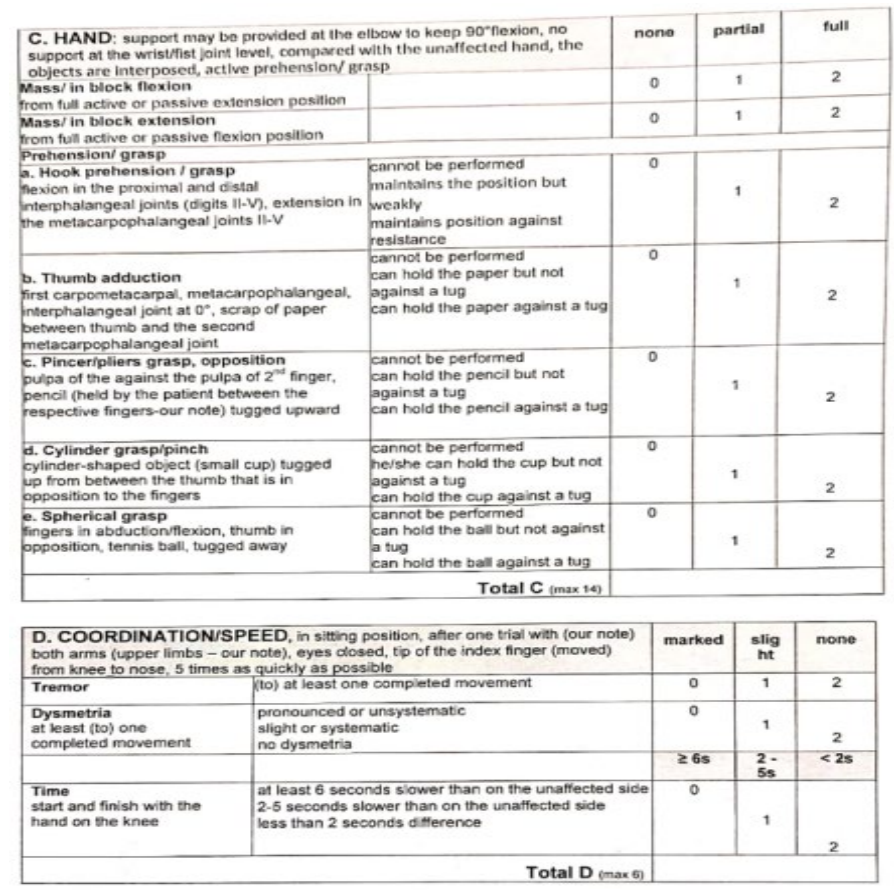

\begin{tabular}{|c|c|c|c|c|c|c|}
\hline \multicolumn{7}{|c|}{\begin{tabular}{|c|c|} 
TOTAL A-D $(\max 66)$ \\
\end{tabular}} \\
\hline \multicolumn{4}{|c|}{$\begin{array}{l}\text { 1. PASSIVE JOINT MOTION, upper extremily, sitting position, } \\
\text { compared to the unaffected side }\end{array}$} & \multicolumn{3}{|c|}{$\begin{array}{l}\text { J. JOINT PAIN during passive motion, } \\
\text { upper extremily }\end{array}$} \\
\hline & \begin{tabular}{|c|} 
only few degrees \\
(less than $10^{0}$ in \\
the shoulder)
\end{tabular} & low & normal & $\begin{array}{c}\text { pronounced pain during } \\
\text { movement or very marked } \\
\text { pain at the end of the } \\
\text { moverement }\end{array}$ & slight & no pain \\
\hline 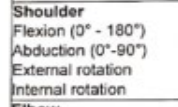 & $\begin{array}{l}0 \\
0 \\
0 \\
0\end{array}$ & $\begin{array}{l}1 \\
1 \\
1 \\
1 \\
\end{array}$ & $\begin{array}{l}2 \\
2 \\
2 \\
2 \\
\end{array}$ & $\begin{array}{l}0 \\
0 \\
0 \\
0\end{array}$ & $\begin{array}{l}1 \\
1 \\
1 \\
1 \\
\end{array}$ & $\begin{array}{l}2 \\
2 \\
2 \\
2 \\
\end{array}$ \\
\hline $\begin{array}{l}\text { Ellow } \\
\text { Flexion } \\
\text { Extension }\end{array}$ & $\stackrel{0}{0}$ & $i$ & $\begin{array}{l}2 \\
2\end{array}$ & $:$ & $i$ & $\begin{array}{l}2 \\
2\end{array}$ \\
\hline $\begin{array}{l}\text { Forearm } \\
\text { Pronation } \\
\text { Supination }\end{array}$ & $\therefore$ & 1 & $\begin{array}{l}2 \\
2\end{array}$ & 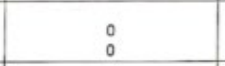 & 1 & $\begin{array}{l}2 \\
2\end{array}$ \\
\hline $\begin{array}{l}\text { Wrist } \\
\text { (fist/ radiocarpal joint) } \\
\text { Flexion } \\
\text { Extension }\end{array}$ & $\begin{array}{l}0 \\
0\end{array}$ & $i$ & $\begin{array}{l}2 \\
2\end{array}$ & : & $i$ & $\begin{array}{l}2 \\
2\end{array}$ \\
\hline $\begin{array}{l}\text { Fingers } \\
\text { Elexion } \\
\text { Extension }\end{array}$ & $: 0$ & 1 & $\begin{array}{l}2 \\
2 \\
\end{array}$ & $\begin{array}{l}0 \\
0\end{array}$ & $\stackrel{1}{1}$ & $\begin{array}{l}2 \\
2\end{array}$ \\
\hline Total $(\max 2$ & & & & Total $(\max 24)$ & & \\
\hline
\end{tabular}

\begin{tabular}{|l|r|}
\hline A. UPPER EXTREMITY & 136 \\
\hline B. WRIST (fistradiocarpal joint) & 110 \\
\hline C. HAND & 114 \\
\hline D. COORDINATION/SPEED & 16 \\
\hline TOTAL A-D (motor function) & 166 \\
\hline
\end{tabular}

\begin{tabular}{|l|r|}
\hline H. SENSATION & 112 \\
\hline I. PASSIVE JOINT MOTION & 124 \\
\hline J. JOINT PAIN & 124 \\
\hline
\end{tabular}

\begin{tabular}{|l|c|c|c|c|}
\hline \begin{tabular}{|l|l|l|} 
H. SENSATION, upper extremity, eyes closed, in \\
comparison to the unaffected side
\end{tabular} & anesthesia & $\begin{array}{c}\text { hypoesthesia or } \\
\text { dysesthesia }\end{array}$ & normal \\
\hline Light touch & $\begin{array}{l}\text { upper arm } \\
\text { forearm, palmary surface of } \\
\text { the hand }\end{array}$ & 0 & 1 & 2 \\
\hline & $\begin{array}{c}\text { less than 3/4 } \\
\text { correct or } \\
\text { absent }\end{array}$ & $\begin{array}{c}\text { 3/4 correct or } \\
\text { considerable } \\
\text { difference }\end{array}$ & $\begin{array}{c}100 \% \text { correct, } \\
\text { little or no } \\
\text { difference }\end{array}$ \\
\hline $\begin{array}{l}\text { Position } \\
\text { slight alterations in in } \\
\text { the position }\end{array}$ & $\begin{array}{l}\text { shoulder } \\
\text { elbow } \\
\text { wrist (fist radiocarpal joint) } \\
\text { thumb (interphalangeal joint) }\end{array}$ & 0 & 1 & 2 \\
\hline & 0 & 1 & 2 \\
\hline
\end{tabular}

Fig. 11. Fugl-Meyer assessment scale translated from Romanian to English (backward)- upper extremity

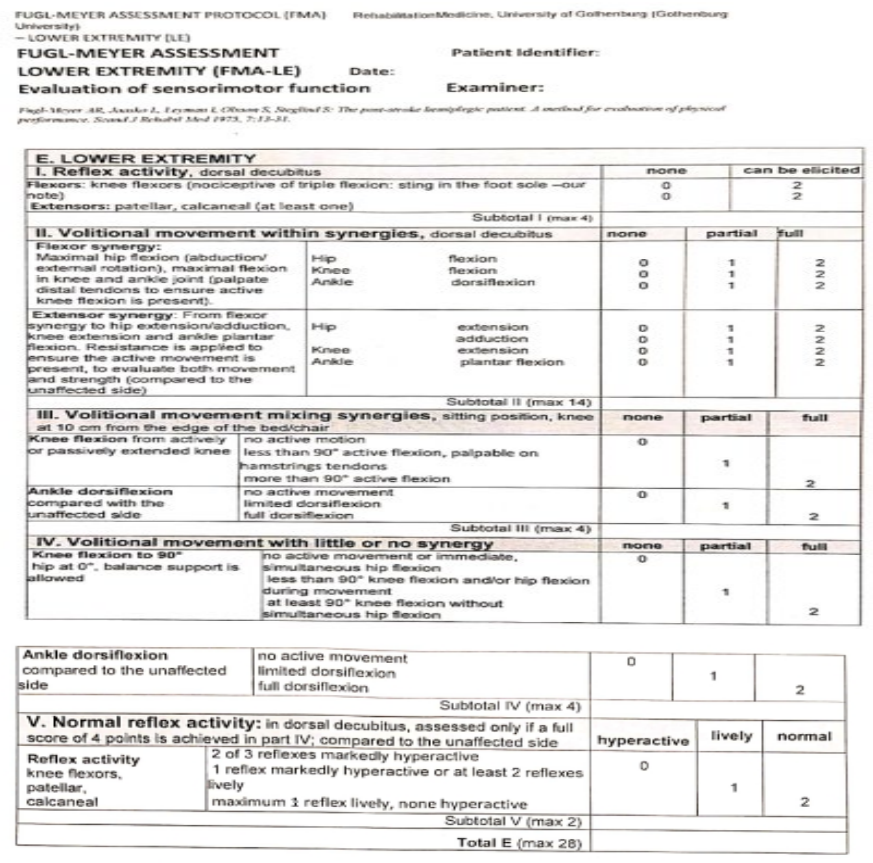

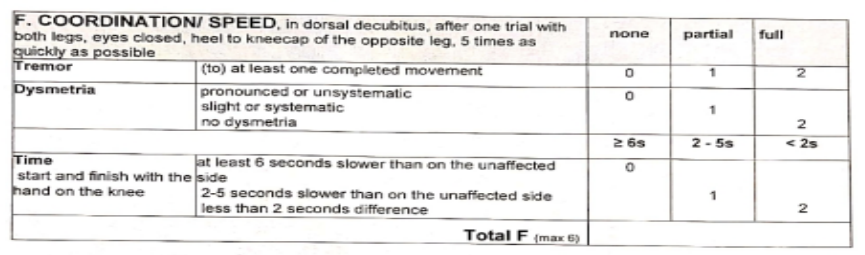

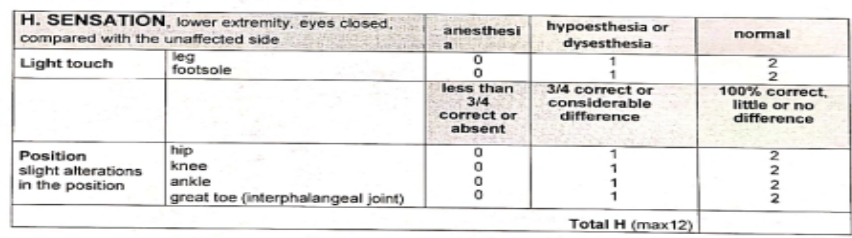

1. PASSIVE JOINT MOTION, lower extremity, dorsal J. JOINT PAIN during passive motion,

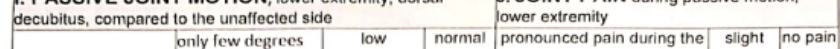

\begin{tabular}{|c|c|c|c|c|c|c|}
\hline \multicolumn{4}{|c|}{ decubitus, compared to the unaffecled side } & \\
\hline & $\begin{array}{l}\text { only lew degrees } \\
\text { (<10 in the hip) }\end{array}$ & low & normal & $\begin{array}{l}\text { pronounced pain during the } \\
\text { movement of very marked } \\
\text { pain at the end of the } \\
\text { movement }\end{array}$ & $\begin{array}{l}\text { slight } \\
\text { pain }\end{array}$ & no pain \\
\hline $\begin{array}{l}\text { Hip } \\
\text { Flexion }\end{array}$ & & & & & & \\
\hline $\mid \begin{array}{l}\text { Flexion } \\
\text { Abduction }\end{array}$ & $\begin{array}{l}0 \\
0\end{array}$ & $i$ & 2 & 0 & 1 & $\begin{array}{l}2 \\
2\end{array}$ \\
\hline External rotation & 0 & 1 & 2 & 0 & 1 & 2 \\
\hline Internal rotation & 0 & 1 & 2 & 0 & 1 & 2 \\
\hline \multicolumn{7}{|l|}{ Knee } \\
\hline $\begin{array}{l}\text { Flexion } \\
\text { Extension }\end{array}$ & 0 & 1 & 2 & 0 & 1 & 2 \\
\hline \multicolumn{7}{|l|}{ Ankle } \\
\hline Dorsiflexion & 0 & 1 & 2 & 0 & 1 & 2 \\
\hline Plantar flexion & 0 & 1 & 2 & 0 & 1 & 2 \\
\hline \multicolumn{7}{|l|}{ Foot } \\
\hline $\begin{array}{l}\text { Pronation } \\
\text { Supination }\end{array}$ & $\begin{array}{l}0 \\
0\end{array}$ & 1 & 2 & 0 & 1 & 2 \\
\hline $\begin{array}{l}\text { Supination } \\
\text { Total } \max 20\end{array}$ & & & & 0 & 1 & 2 \\
\hline
\end{tabular}

\section{\begin{tabular}{|l|r|}
\hline E. LOWER EXTREMITY & 128 \\
\hline F. COORDINATION/SPEED & 16 \\
\hline
\end{tabular} \\ \begin{tabular}{|l|r|}
\hline F. COORDINATION/SPEED & 16 \\
\hline TOTAL E-F (motor function) & 134 \\
\hline
\end{tabular}}

\begin{tabular}{l|r|}
\hline H. SENSATION & 112 \\
\hline I. PASSIVE JOINT MOTION & 120 \\
\hline J. JOINT PAIN & 120 \\
\hline
\end{tabular}

Fig.12. Fugl-Meyer assessment scale translated from Romanian to English (backward)- lower extremity Pending on the outcome of the above mentioned correspondence, hopefully we could succeed to enhance FMA applying, but in a reasonable time framing within clinical rhythm and considering the unfortunate actual period of COVID-19 pandemic. 